\title{
A secondary pseudo-ainhum associated to spinocellular carcinoma
}

Keywords: pseudo-ainhum, amputation, lesions, lymph node, bone resorption, spinocellular carcinoma

\section{Introduction}

Pseudoainhum is a rare manifestation of band like constriction progressing into an auto amputation in the affected part. ${ }^{1}$ In contrast to ainhum disease pseudoainhum occurs as a result of an identifiable or associated disease process and is independent of skin colour. It is also not limited to the digits and can occur on the trunk of the body. ${ }^{2}$ We report for the first time a pseudoainhum disease associated with a spinocellular carcinoma of the leg.

\section{Case report}

A 40-years-old man attended the dermatology department in Hassan II hospital in Fez with a 20years history of non-healing ulcer on the back foot. It wasn't associated with pain. There was no history of diabetes mellitus, and he was a non-smoker. He was mobile and lived independently. The evolution was marked by the extension of the ulcer, and progressed to substance loose leading to spontaneous amputation of more than $1 / 3^{\text {rd }}$ of the leg. Examination revealed an ulcerative budding stump of the leg (Figure 1). A biopsy was done, and the anatomy pathology revealed a verrucous spinocellular carcinoma. Leg radiography showed a complete destruction of the fibula and tibia and resorption of the remaining bones of the leg. An ultrasound of ganglionic areas and a thoraco-abdominal CT scan didn't reveal any abnormalities (Figure2). After consultation with the traumatologist, the patient had undergone transtibial amputations with lymph node dissection. The anatomy pathology concluded to spinocellular carcinoma. The patient healed and is still alive 3years after the amputation. He didn't present any similar lesions.

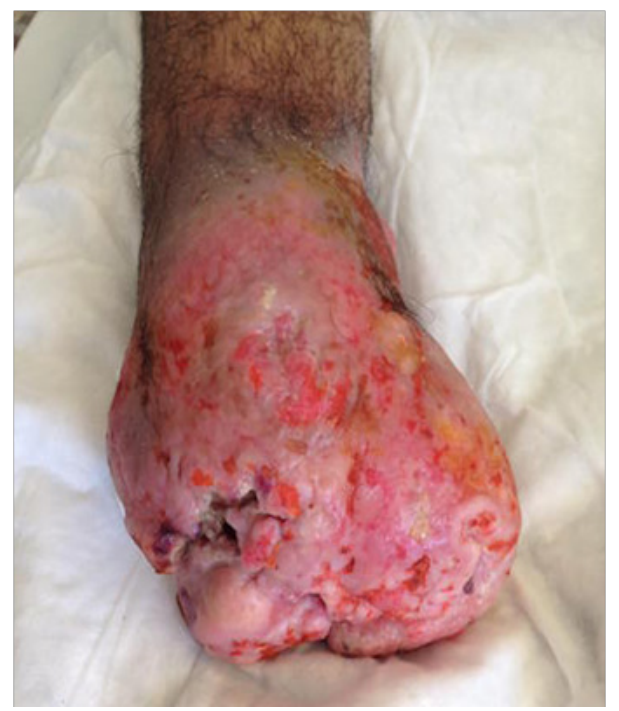

Figure I Ulcerative budding stump.
Volume 2 Issue 4 - 2018

\section{Nassiri A,' Moustaide K,' Gallouj S,' Mernissi Fz,' Abdelhafid El Marfi, ${ }^{2}$ Abdelmajid El Mrini² \\ 'Department of dermatology, Hassan II hospital, Moroco \\ ${ }^{2}$ Department of traumatology, Hassan II hospital, Morocco}

Correspondence: A Nassiri, department of dermatology, hassan II hospital, road of Sidi hrazem, FES, Morocco, Email aichanassiri6@gmail.com

Received: February 14, 2018| Published: July 06, 2018
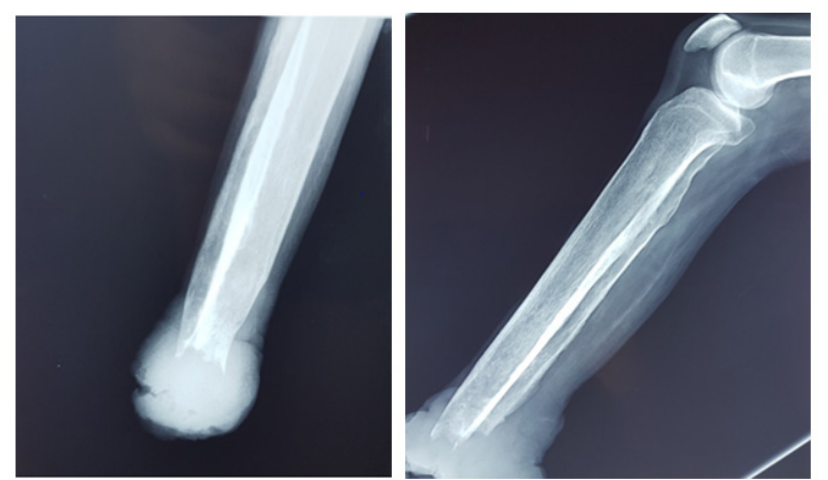

Figure 2 Bone destruction and resorption.

\section{Discussion}

First described by Messum in 1821, as constricting bands, the pseudoainhum disease wasn't well distinguished from ainhum disease until 1953 by Neumann. ${ }^{3}$ He also confirmed that amniotic band lesions are different from pseudoainhum. Pseudoainhum has been divided into two sections: primary pseudoainhum and secondary pseudoainhum. Secondary pseudoainhum is an auto-amputation that develops later in life and is a result of an identifiable or associated process. It is independent of skin colour and it is not restricted to the digits and limbs. ${ }^{4}$

Pseudoainhum is under reported in literature, that may be secondary to the lack of clarity of the terminology. ${ }^{5}$ Many reports of secondary pseudoainhum are presented in order to clarify the large panel of association such as papillon-lefevre syndrome, psoriasis $^{6}$ and scleroderma. ${ }^{7}$ Many reports tried to identify aetiology, considering different theories. The most aetiology overlooked causes are: mechanical, ${ }^{2}$ genetic predisposition, ${ }^{8}$ vascular abnormalities. ${ }^{9}$ Pseudoainhum was also described in association with breast cancer. ${ }^{10}$ We are reporting the first case of pseudoainhum disease associated with spinocellular carcinoma of the leg. Pseudoainhum may progress in weeks, as it can do it in years as in our case. ${ }^{2}$ It can begin as a superficial lesion of the skin or as a deep groove reaching the bone. ${ }^{11}$ Extremities are the most described areas to be touched because they are hypo-perfused as well as hyperkeratotic regions. ${ }^{12}$ Pain is not 
always described, bone resorption may be seen on radiography. ${ }^{12}$

The diagnosis of pseudoainhum disease is based on medical history and physical examination. No laboratory exams can be done to confirm the diagnosis.

\section{Primary and secondary pseudoainhum progress over 4stages}

a. Formation of a groove or fissure with a hyperkeratotic callus.

b. Arterial narrowing with eventual cessation of blood flow and oedema formation.

c. Separation of the bones from the joint with a hyper mobile digit.

d. Blood-less auto-amputation. ${ }^{13}$

Unfortunately, until now, there is no consensus for the management of pseudoainhum. Many attempts reported liver oil and nicotinic acid. Surgery and etretinate had also been reported..$^{14,15}$

\section{Conclusion}

Secondary pseudoainhum is a rare disease but it can be very traumatic and painful, which can impact the quality of life of the patient. Many possible syndromes or diseases may be associated with Secondary pseudoainhum. A detailed work-up is needed to clarify all possible associations. If done, the therapeutic management might be improved.

\section{Acknowledgments}

None

\section{Conflict of interest}

The author declared that there are no conflicts of interest.

\section{References}

1. Davis BS, Harris S, Forman MD. Secondary pseudoainhum in a patient with Turner syndrome. J Am Osteopath Assoc. 2014;114(10):806-808.

2. Rashid RM, Cowan E, Abbasi SA, et al. Destructive deformation of the digits with auto-amputation: a review of pseudo-ainhum. J Eur Acad Dermatol Venereol. 2007;21(6):732-737.

3. Neumann A. Pseudoainhum: report of congenital case involving several fingers in the left wrist. Arch Derm Syphilol. 1953;68(4):421-427.
4. Olivieri I, Piccirillo A, Scarano E, et al. Dactylolysis spontanea or ainhum involving the big toe. J Rheumatol. 2005;32(12):2437-2439.

5. Daccarett M, Espinosa G, Rahimi F, et al. Ainhum (dactylolysis spontanea): a radiological survey of 6000 patients. J Foot Ankle Surg. 2002;41(6):372378.

6. Mc Laurin CI. Psoriasis presenting with pseudoainhum. J Am Acad Dermatol. 1982;7(1):130-132.

7. Park BS, Cho KH, Youn JI, et al. Pseudoainhum associated with linear scleroderma. Arch Dermatol. 1996;132(12):1520-1521.

8. O'Driscoll J, Muston GC, Mc Grath JA, et al. A recurrent mutation in the loricrin gene underlies the ichthyotic variant of Vohwinkel syndrome. Clin Exp Dermatol. 2002;279(3):243-246.

9. Graham RM, James MP. Pseudoainhum, angiodysplasia and focal acral hyperkeratosis. J R Soc Med. 1985;78(Suppl 11):13-15.

10. Wollina U, Graefe T, Oelzner P, et al. Pseudoainhum of all fingers associated with Reynolds' syndrome and breast cancer: report of a case and review of the literature. J Am Acad Dermatol. 2001;44(2 Suppl.):381-384.

11. Raque CJ, Stein KM, Lane JM. Pseudoainhum constricting bands of the extremities. Arch Dermatol. 1972;105(3):434-438.

12. Genakos JJ, Cocores JA, Terris A. Ainhum (dactylosis spontanea): report of a bilateral case and literature review. J Am Podiatr Med Assoc. 1986;76(12):676-680.

13. Browne SG. Ainhum. Int J Dermatol. 1976;15(5):348-350.

14. Kandhari KC, Manchanda SS. Ainhum and pseudoainhum. Report of three cases. Dermatol Trop Ecol Geogr. 1963;19:6-10.

15. Poulin Y, Perry HO, Muller SA. Olmsted syndrome - congenital palmoplantar and periorificial keratoderma. J Am Acad Dermatol. 1984;10(4):600-610. 